\title{
Dermatologia e tatuaggi
}

\author{
Marina Conese ', Valerio Cirfera ${ }^{2}$
}

Specialista in Dermatologia e Venereologia, Bari

Specialista in Dermatologia e Venereologia. Coordinatore Nazionale AIDA-GIDeL,

Responsabile Dermatologia Scienze Medico-Legali, Università degli Studi di Siena [Dir.: Prof. Cosimo Loré]

\begin{abstract}
Medical-legal aspects of tatooing are of topical interest, concerning many matters of discussion. Tattooing is an invasive procedure and can be associated with infectious and noninfectious complications, expecially when done by inexperienced. This article emphasizes the need for legal regulation of tattooing.
\end{abstract}

Keywords: tattooing, medical complication, liability, legal aspects

Dermatology and tattooing

Pratica Medica \& Aspetti Legali 2008; 2(2): 107-111

\section{INTRODUZIONE}

Il tatuaggio (vocabolo proveniente dal linguaggio di Tahiti "tatau" o "tatu") è il risultato della penetrazione di granuli di sostanze insolubili e colorate nel derma con esito in discromie (macchie) indelebili. Può essere "accidentale" o provocato volontariamente sulla pelle a scopo decorativo, o per camouflage (eseguito per un fine cosmetico-correttivo), come avviene per i nevi flammei o per esempio in pazienti portatori di vitiligine. In campo medico-legale ben noti risultano essere i tatuaggi accidentali, formatisi per colpi di arma da fuoco esplosi a distanza molto ravvicinata. Esiste poi un terzo gruppo di tatuaggi, oltre a quelli accidentali e volontari, costituito dalle "dermato-coniosi" riconducibili a cause professionali (fuochisti, minatori, metallurgici).

A seconda del colore della pelle il tatuaggio si può realizzare in due modi differenti: se essa è fortemente pigmentata si realizza la scarificazione con esito in cheloidi; se invece la pigmentazione è scarsa ha luogo il tatuaggio propriamente detto. Una volta disegnato il motivo sulla pelle si procede ad una serie di punture molto ravvicinate le une alle altre mediante un ago intriso di colore prescelto. I professionisti del tatuaggio si servono di un ago elettrico che realizza il disegno sulla cute introducendo automaticamente particelle di pigmento nello strato più profondo del derma. Ne consegue che la profondità della deposizione di pigmento nel derma è diversa a seconda di chi ha praticato il tatuaggio. Risulta essere infatti superficiale ed uniforme se il tatuaggio è stato eseguito da un professionista, più profondo ed irregolarmente distribuito se commissionato a un amatore o a una persona poco esperta.

I pigmenti più comunemente usati sono carbone per il blu-nero, cinabro e tinture vegetali per il rosso, cobalto per il blu chiaro, cromo per il verde, cadmio per il giallo e silice dell'ocra per i colori bruni.

\section{ASPETTI MEDICO-LEGALI DEL TATUAGGIO}

I tatuaggi, costituendo dei segni indelebili della pelle, sono utilizzati come veri e propri markers 
per l'identificazione personale, assumendo un'importanza notevole in medicina forense specialmente in casi di sconosciuta o incerta identità. In linea del tutto generale può però aversi una modificazione dell'intensità della tinta, direttamente proporzionale al tempo trascorso dal momento in cui è stato eseguito. Purtroppo a questa regola vi sono eccezioni, in quanto i tatuaggi "sbiaditi" possono essere stati eseguiti da poco tempo e viceversa si possono mostrare "freschi" (cioè che conservano la stessa intensità del colore originario) quelli eseguiti parecchi anni prima. In campo penale può avere importanza cercare di datare un tatuaggio, cioè risalire all'epoca in cui questo fu eseguito. In linea di massima si è detto che più tempo trascorre più questo diventa sfumato. Concorrono diversi fattori a realizzare ciò. In prima istanza si fa menzione del fatto che le particelle di pigmento sono fagocitate dal sistema macrofagico dermico, il quale lentamente ma in maniera costante drena il pigmento fagocitato ai linfonodi loco-regionali. Ciò contribuirebbe a sbiadire il tatuaggio. Anche l'esposizione continua e prolungata ai raggi ultravioletti accelererebbe il suo processo di degradazione.

\section{CASO CLINICO}

Quesito del Giudice e scopo dell'incarico di Consulenza Tecnica d'Ufficio: accertare il tempo strettamente necessario per la esecuzione dei tatuaggi di cui è portatore l'imputato.

Il paziente è un detenuto di 32 anni, coltivatore diretto. Ai fini della presente indagine non si registra nulla di rilievo nell'ambito dell'anamnesi patologica remota e prossima. In merito all'epoca in cui sono stati realizzati i tatuaggi, oggetto di indagine, l'uomo sostiene che il primo fu realizzato nel 1980 e gli altri nel 1985. Riferisce inoltre che gli autori del tatuaggio sarebbero state due differenti persone che si avvalsero di un ago da cucito e di inchiostro di china, in quanto i tatuaggi furono effettuati in regime di detenzione. Sottolinea inoltre di aver accusato dolore durante la loro esecuzione e che comparve modesto grado di arrossamento ed edema. Precisa che non fu possibile completare in un'unica seduta la realizzazione del tatuaggio più esteso, a causa del considerevole gonfiore e dolenzia locale, ma ne furono necessarie due, a distanza di una decina di giorni circa l'una dall'altra. Al contrario l'esecuzione degli altri tatuaggi di più modeste dimensioni richiese qualche ora ciascuno.

All'esame obiettivo il paziente si presenta in buone condizioni generali di nutrizione e sanguificazione. La cute è normotrofica e normoelastica; il pannicolo adiposo è normalmente rappresentato e distribuito secondo sesso ed età. Sulla superficie cutanea del soggetto sono presenti complessivamente 11 tatuaggi, distribuiti nelle seguenti regioni:

- avambraccio destro: sulla superficie volare a livello del polso, piccolo tatuaggio monocromatico, di colore blu scuro, costituito dal numero 6 sottolineato e dalla dicitura "MIA" posta inferiormente;

- arto inferiore destro: sono presenti 7 tatuaggi ai seguenti livelli:

- coscia: sono presenti due tatuaggi. Il primo, sulla superficie antero-laterale, è bicromatico e interessa circa $11 \times 9 \mathrm{~cm}$ della superficie cutanea. Raffigura un disegno a ghirlanda costituito da tre fiori collegati fra loro a costituire una ghirlanda, la dicitura "AMO MAMMA" e un disegno a mo' di asterisco inscritto nella cute delimitata dalla ghirlanda. I colori adoperati sono il blu scuro e il rosso. Il secondo è sulla superficie anteriore; è un tatuaggio monocromatico (blu scuro) raffigurante: una croce, ricavata da numerosi punti distanti tra loro $0,5 \mathrm{~mm}$; una lettera $\mathrm{E}$ capovolta e 5 punti che rappresentano il 5 di un dado da gioco;

- gamba: superficie laterale 3 medio: due tatuaggi. Il primo monocromatico, di colore blu scuro, è costituito da 5 punti che rappresentano il 5 di un dado da gioco. Il secondo, bicromatico, di colore blu scuro e rosso, raffigura i contorni, il seme e la figura di 4 carte da gioco affiancate;

- piede: superficie dorsale con 3 tatuaggi raffiguranti due labbra, 3 segni puntiformi e 5 segni puntiformi (simbolo 5 del dado da gioco); i colori utilizzati sono il blu scuro e il rosso;

- arto inferiore sinistro:

- coscia: a livello della superficie antero-laterale si apprezza un vasto tatuaggio tricromatico, di colore blu, verde e rosso sfumato, raffigurante un drago alato, lungo circa $23 \mathrm{~cm}$ (Figura 1);

- gamba: a livello della superficie posteriore del $3^{\circ}$ medio del polpaccio si apprezza un piccolo tatuaggio bicromatico di colore blu scuro e rosso sfumato, raffigurante un piccolo drago;

- piede: sulla superficie dorsale è presente un tatuaggio monocromatico, blu scuro, raffigurante un volatile (colomba o rondine).

\section{VALUTAZIONE DEL CASO}

In occasione della visita dermatologica sono stati obiettivati 11 tatuaggi, localizzati in diverse aree corporee. 
In letteratura si segnalano fondamentalmente due metodiche per la realizzazione del tatuaggio: quello "americano" e quello "europeo" $[1,2]$.

Il primo metodo, americano, utilizza un dispositivo azionato elettricamente in cui la parte terminale di un tubo, costituita da un ago, è bagnata da un po' di inchiostro; esso, muovendosi in ogni piccola porzione di cute e spingendosi delicatamente in profondità dall'epidermide al derma, deposita del pigmento insolubile. Quando l'inchiostro inizia a diffondersi, l'estremità del dispositivo viene intrisa nuovamente e il processo ripetuto. L'eccesso di inchiostro è asciugato e portato via da un tessuto assorbente che rimuove anche le piccole particelle di sangue che si formano dalle punture parcellari eseguite sulla cute.

Nel metodo europeo l'inchiostro è previamente spalmato su ogni piccola area che deve essere tatuata; successivamente l'ago è azionato facendo penetrare così i pigmenti insolubili nel derma.

In linea generale, mentre la tecnica utilizzata da "professionisti del tatuaggio" si avvale di un idoneo apparecchio (ago elettrico) che realizza il disegno sulla cute introducendo automaticamente particelle di pigmento nello strato più profondo del derma, i dilettanti iniettano mediante sottili strumenti appuntiti piccole particelle di nerofumo o inchiostro di china nella pelle, senza cioè l'ausilio di uno strumento speciale. A riguardo è opportuno precisare che la profondità della deposizione di pigmento nel derma è diversa a seconda della tecnica utilizzata da chi ha praticato il tatuaggio. Il pigmento infatti risulta essere più superficiale e uniforme se il tatuaggio è stato eseguito da un professionista, più profondo e irregolarmente distribuito se realizzato da un dilettante o da persona poco esperta.

Per una valutazione dei tempi di esecuzione di un tatuaggio bisogna tenere conto di alcune variabili oggettive, che dipendono essenzialmente da morfologia, dimensioni del disegno da realizzare e capacità professionali, nonché di variabili soggettive, che sono correlate alla risposta individuale e locale al trattamento. In linea del tutto generale si può affermare che tatuaggi di piccolissime dimensioni e monocromatici possono essere completati in meno di un'ora da un esperto; tatuaggi che ricoprono estesi lembi di cute e policromatici, richiedono più tempo, settimane $o$ mesi. Ma al di là delle capacità professionali di chi esegue il tatuaggio, occorre sottolineare che se il disegno per le sue caratteristiche morfologiche e cromatiche non può essere completato in una sola seduta, è necessario un congruo intervallo di tempo prima di sottoporre la cute a nuove infissioni. È importante ricordare che tale periodo di riposo, richiesto per poter riprendere il trattamento, dipende dalla inevitabile reazione infiammatoria dermica, la cui regressione si verifica all'incirca in 5-10 giorni [3], arco di tempo variabile

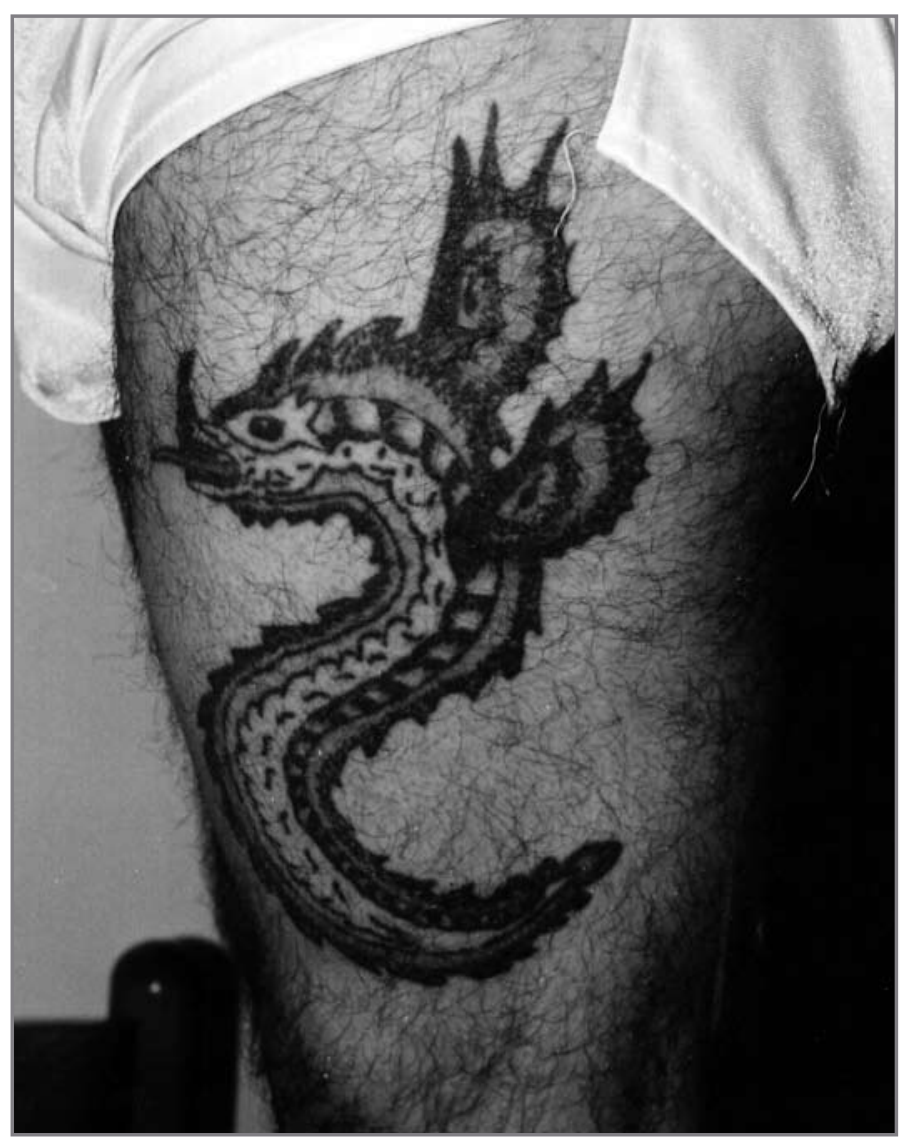

Figura 1. Tatuaggio a livello della coscia sinistra

in rapporto alla tecnica utilizzata, alla complessità del disegno e alla reazione individuale. Questa reazione locale rende impossibile ogni manovra di ripasso sulla zona sia per lo stato di edema infiammatorio, che non consentirebbe una corretta esecuzione del tatuaggio, sia e soprattutto per il dolore che si accompagna all'infiammazione. Ovviamente l'uso di antibiotici e/o antiflogistici potrebbe accelerare il processo di risoluzione dell'infiammazione con la restitutio ad integrum del tessuto leso.

Oltre alla valutazione di queste variabili locali, che dipendono dal processo flogistico, sono da considerare nella valutazione dei tempi di esecuzione di tatuaggi più complessi le variabili individuali: capacità di sopportazione del dolore e sedi corporee in cui viene praticato il tatuaggio, in quanto alcune zone sono più sensibili agli stimoli dolorifici di altre.

Inoltre appare evidente che i vari tatuaggi manifestano una differente preparazione tecnica da parte dell'operatore in funzione del diverso grado di definizione dei vari disegni oltre che della complessità dei vari soggetti realizzati graficamente. Pertanto può ritenersi verosimile l'attribuzione dei tatuaggi ad almeno due diversi operatori: tra questi, ad un operatore più esperto la realizzazione dei tatuaggi raffiguranti il drago alato, la ghirlanda e il piccolo serpente. 


\section{RISPOSTA AL QUESITO E CONCLUSIONI}

Sulla base di queste indicazioni, analizzando i tatuaggi obiettivati sul soggetto in esame e posto anche il numero complessivo degli stessi [1], si può ritenere del tutto verosimile che per la loro esecuzione sia stata richiesta più di una seduta. In particolare per i tatuaggi monocromatici si può ritenere congruo valutare i tempi medi di esecuzione in un periodo variabile per ciascuno di essi tra 1 e 3 ore circa.

Per i tatuaggi bicromatici, tenendo presente la complicatezza del disegno effettuato e l'uso di due colori, i tempi di esecuzione devono ritenersi variabili soprattutto in rapporto alle dimensioni come segue:

- superficie antero-laterale coscia dx (ghirlanda AMO MAMMA): 5-6 ore circa;

- $\quad$ superficie dorsale piede dx (due labbra): 1-2 ore circa;

- superficie posteriore gamba sx (piccolo drago): 3-4 ore circa.

Per l'unico tatuaggio tricromatico, posto alla superficie antero-laterale dell'arto inferiore sx, orientativamente il tempo necessario può essere valutato da un minimo di una seduta di 9-10 ore circa ad un massimo di 2 sedute intervallate di circa una settimana una dall'altra. Infine è sempre auspicabile ed eticamente doveroso un corretto operato del consulente chiamato ad esprimere il proprio giudizio e nei casi in cui come questo le indicazioni fornite dal consulente sono da ritenersi approssimative (in quanto vi è l'impossibilità di stabilire con certezza la tecnica utilizzata per l'esecuzione dei tatuaggi e di valutare con maggiore precisione la reazione individuale di tipo infiammatorio), deve comunque ritenersi un successo professionale in quanto stimolo per il medico all'approfondimento di alcune tematiche specifiche.

\section{RIFLESSIONI CONCLUSIVE}

Il caso è molto interessante perché, oltre alla valenza culturale e nello stesso tempo pratica del quesito posto dal Giudice, riporta all'attenzione una problematica di interesse socio-culturale, legale e sanitario, per i motivi simbolici che spingono all'esecuzione di essa, per la qualifica dell'operatore, l'autorizzazione ad eseguirli, in mancanza della quale si palesa il reato di abusivismo di professione da parte di soggetti spesso improvvisati e non competenti e infine per l'ambito strutturale dove si esegue e per i rischi sulla salute, che talvolta realizzano dei veri e propri danni gravi alla persona, ovvero lesioni attraverso delle condotte che possono essere ritenute colpose.

Il fenomeno, sotto questi aspetti negativi, è ben conosciuto in certi ambienti sociali o comunitari, come appunto quelli carcerari, dove la pratica del tatuaggio è molto diffusa, ma illecita, eccezion fatta per i casi inseriti in iniziative sociali di prevenzione del rischio, controllate e autorizzate [4]. Quasi sempre in ambienti non protetti, la pratica del tatuaggio viene effettuata senza il rispetto delle più elementari cognizioni e requisiti tecnici e di igiene, per cui è alto il rischio di allergie, infezioni, specie epatiti, contagi, soprattutto per messa in pratica di procedure rudimentali al limite dell'autolesionismo se fatte eseguire sulla propria persona da non professionisti che non conoscono bene la tecnica precisa, i tempi e le modalità esecutive, di solito messe in atto con mezzi approssimativi e pericolosi.

L'operatore autorizzato, invece, è ben conscio che dovrà garantire i livelli raccomandati di igiene operativa, così come è previsto anche per la pratica del piercing dalle linee guida emanate dal Consiglio Superiore di Sanità nel 1998 e dalle più recenti disposizioni a livello regionale [5], informare adeguatamente il suo "cliente" sulla metodica, sui suoi possibili rischi e sulla irreversibilità dell'impianto, evenienza che presuppone l'esclusione di allergie verso quel o quei pigmenti. Questa e altre problematiche correlate fanno riflettere non poco sui motivi per cui la legge non considera il tatuaggio e il piercing atti medici, ovvero attività invasive sul corpo umano, ma solo attività estetico-decorative; non sussiste, quindi, l'obbligatorietà di una visita medica preventiva o di controlli sanitari successivi all'impianto, a meno che non accada una complicanza. Si riporta il relativo richiamo giuridico: «ai fini del delitto di cui all'art. 348 del codice penale, l'attività di piercing e tatuaggio, non persegue finalità terapeutiche, ossia di diagnosi, profilassi e cura di eventi morbosi, bensì solo decorative. Conseguentemente essa non rientra nell'ambito della professione sanitaria e delle relative arti ausiliarie, per le quali si richiede la speciale abilitazione dello stato (fattispecie in tema di sequestro preventivo di un laboratorio per il tatuaggio)» [6].

Tale osservazione critica non può che portare a ribadire con forza l'esigenza e/o la necessità della fattiva e auspicabile collaborazione tra operatori autorizzati e Sanitari [7]. 


\section{BIBLIOGRAFIA}

1. Sperry K. Tattoos and tattooing. Part I: history and methodology. Am J Forensic Med Pathol 1991; 12: 313-9

2. Sperry K. Tattoos and tattooing. Part II: gross pathology, histopathology, medical complications, and applications. Am J Forensic Med Pathol 1992; 13: 7-17

3. Betz P. Histological and enzyme histochemical parameters for the age estimation of human skin wounds. Int J Leg Med 1994; 107: $60-8$

4. Progetto carcere Ser.T. di Venezia. Disponibile su: http:/www.ristretti.it/areestudio/droghe/progetti/riduzione.htm

5. Decreto Direzione Generale Sanità Regione Lombardia n. 6932 del 27 aprile 2004 in materia di "Linee Guida per l'esercizio delle attività di tatuaggio e/o piercing"

6. Cassazione penale, sez. VI, sentenza n. 524 del 21 maggio 1996

7. Cirfera V. Riflessioni sul piercing. Il Dermatologo 2007; 24: 15 